\title{
On metric-preserving functions and fixed point theorems
}

\author{
Prapanpong Pongsriiam ${ }^{1 *}$ and Imchit Termwuttipong ${ }^{2}$
}

"Correspondence:

prapanpong@gmail.com

${ }^{1}$ Department of Mathematics,

Faculty of Science, Silpakorn

University, Nakhon Pathom, 73000,

Thailand

Full list of author information is

available at the end of the article

\begin{abstract}
Kirk and Shahzad have recently given, in this journal, fixed point theorems concerning local radial contractions and metric transforms. In this article, we replace the metric transforms by metric-preserving functions. This in turn gives several extensions of the main results given by Kirk and Shahzad. Several examples are given. The fixed point sets of metric transforms and metric-preserving functions are also investigated.
\end{abstract}

Keywords: metric-preserving function; metric transform; local radial contraction; rectifiably pathwise connected space; uniform local multivalued contraction

\section{Introduction}

The concept of metric transforms is introduced by Blumenthal [1,2] in 1936 while the concept of metric-preserving functions seems to be introduced by Wilson [3] in 1935 and is investigated in detail by many authors [4-20]. Recently, Petruşel et al. [14] have shown the role of equivalent metrics and metric-preserving functions in fixed point theory. In addition, Kirk and Shahzad [21] have given results concerning metric transforms and fixed point theorems. Their main results are as follows:

Theorem 1 (Kirk and Shahzad [21, Theorem 2.2]) Let $(X, d)$ be a metric space and $g: X \rightarrow X$. Suppose there exist a metric transform $\phi$ on $X$ and a number $k \in(0,1)$ such that the following conditions hold:

(a) For each $x \in X$ there exists $\varepsilon_{x}>0$ such that for every $u \in X$

$$
d(x, u)<\varepsilon \quad \Rightarrow \quad(\phi \circ d)(g(x), g(u)) \leq k d(x, u) .
$$

(b) There exists $c \in(0,1)$ such that for all $t>0$ sufficiently small

$$
k t \leq \phi(c t) .
$$

Then $g$ is a local radial contraction on $(X, d)$.

Theorem 2 (Kirk and Shahzad [21, Theorem 2.3]) Suppose, in addition to the assumptions in Theorem 1, $X$ is complete and rectifiably pathwise connected. Then $g$ has a unique fixed point $x_{0}$, and $\lim _{n \rightarrow \infty} g^{n}(x)=x_{0}$ for each $x \in X$.

2014 Pongsriiam and Termwuttipong; licensee Springer. This is an Open Access article distributed under the terms of the Creative Commons Attribution License (http://creativecommons.org/licenses/by/2.0), which permits unrestricted use, distribution, and reproduction in any medium, provided the original work is properly cited. 
Our purpose is to show that the metric transform $\phi$ in Theorem 1 can be replaced by a metric-preserving function. This in turn gives extensions to the main results given by Kirk and Shahzad in [21, Theorem 2.2, Theorem 2.3, Theorem 2.8, Theorem 3.4, and Theorem 3.6]. Now let us recall some basic definitions that will be used throughout this article.

Definition 3 Let $f:[0, \infty) \rightarrow[0, \infty)$. Then

(i) $f$ is said to be a metric transform if $f(0)=0, f$ is strictly increasing on $[0, \infty)$, and $f$ is concave on $[0, \infty)$,

(ii) $f$ is said to be a metric-preserving function if for all metric spaces $(X, d), f \circ d$ is a metric on $X$,

(iii) $f$ is said to be amenable if $f^{-1}(\{0\})=\{0\}$,

(iv) $f$ is said to be tightly bounded if there exists $u>0$ such that $f(x) \in[u, 2 u]$ for all $x>0$,

(v) $f$ is said to be subadditive if $f(a+b) \leq f(a)+f(b)$ for all $a, b \in[0, \infty)$.

Definition 4 Let $(X, d)$ be a metric space and $g: X \rightarrow X$. Then $g$ is said to be a local radial contraction if there exists $k \in(0,1)$ such that for each $x \in X$, there exists $\varepsilon>0$ such that for every $u \in X$,

$$
d(x, u)<\varepsilon \quad \Rightarrow \quad d(g(x), g(u)) \leq k d(x, u) .
$$

Definition 5 Let $(X, d)$ be a metric space and $\gamma$ be a path in $X$, that is, a continuous map $\gamma:[a, b] \rightarrow X$. A partition $Y$ of $[a, b]$ is a finite collection of points $Y=\left\{y_{0}, \ldots, y_{N}\right\}$ such that $a=y_{0} \leq y_{1} \leq y_{2} \leq \cdots \leq y_{N}=b$. The supremum of the sums

$$
\sum Y=\sum_{i=1}^{N} d\left(\gamma\left(y_{i-1}\right), \gamma\left(y_{i}\right)\right)
$$

over all the partitions $Y$ of $[a, b]$ is called the length of $\gamma$. A path is said to be rectifiable if its length is finite. A metric space is said to be rectifiably pathwise connected if each two points of $X$ can be joined by a rectifiable path.

We will give some auxiliary results in Section 2 . Then we will give the results concerning metric-preserving functions, local radial contractions, and uniform local multivalued contractions in Section 3 and Section 4. Finally, we investigate the fixed point sets of metric transforms and metric-preserving functions in Section 5.

\section{Lemmas}

We need to use some properties of metric-preserving functions and some fixed point theorems. We give them in this section for the convenience of the reader. For more details of the metric-preserving functions, we refer the reader to $[6,8,10]$.

Lemma 6 Let $f:[0, \infty) \rightarrow[0, \infty)$. Then

(i) iff is metric preserving, then $f$ is amenable,

(ii) iff is amenable and concave, then $f$ is metric preserving. 
Proof The proof of (i) is easily obtained; see for example, in [5, Lemma 2.3]. The proof of (ii) is given in [5, Proposition 1.2] and [8, p.13]. See also [4, Proposition 2] and [6, p.311].

Lemma 7 Let $f:[0, \infty) \rightarrow[0, \infty)$. Iff is amenable, subadditive, and increasing, then $f$ is metric preserving.

Proof The proof can be found in [5, Proposition 1.1], [6, Proposition 2.3], and [8, p.9].

Lemma 8 Iff $:[0, \infty) \rightarrow[0, \infty)$ is amenable and tightly bounded, then $f$ is metric preserving.

Proof The proof is given in [5, Proposition 1.3], [6, Proposition 2.8], and [8, p.17].

Lemma 9 Iff is metric preserving and $0 \leq a \leq 2 b$, then $f(a) \leq 2 f(b)$.

Proof The proof is given in [5, Lemma 2.5], and [8, p.16].

For a metric-preserving function $f$, let $K_{f}$ denote the set

$$
K_{f}=\{k>0 \mid f(x) \leq k x \text { for all } x \geq 0\} .
$$

Recall also that we define inf $\emptyset=+\infty$. Then we have the following result.

Lemma 10 Let $f:[0, \infty) \rightarrow[0, \infty)$ be metric preserving. Then $f^{\prime}(0)=\inf K_{f}$. In particular, $f^{\prime}(0)$ always exists in $\mathbb{R} \cup\{+\infty\}$ and

(i) $f^{\prime}(0)<+\infty$ if and only if $K_{f} \neq \emptyset$, and

(ii) $f^{\prime}(0)=+\infty$ if and only if $K_{f}=\emptyset$.

Proof The proof can be found in [4, Theorem 2], [6, Theorem 4.4], and [8, pp.37-39].

The next lemma is probably well known but we give a proof here for completeness.

Lemma 11 If $f:[0, \infty) \rightarrow[0, \infty)$ is amenable and concave, then the function $x \mapsto \frac{f(x)}{x}$ is decreasing on $(0, \infty)$

Proof Let $a, b \in(0, \infty)$ and $a<b$. Since $f$ is concave, we obtain

$$
f(a)=f\left(\left(1-\frac{a}{b}\right)(0)+\left(\frac{a}{b}\right)(b)\right) \geq\left(1-\frac{a}{b}\right) f(0)+\frac{a}{b} f(b)=\frac{a}{b} f(b) .
$$

Therefore $\frac{f(a)}{a} \geq \frac{f(b)}{b}$, as desired.

Lemma 12 (Pokorný $[16])$ Let $f:[0, \infty) \rightarrow[0, \infty)$. Assume that $f$ is amenable and there is a periodic function $g$ such that $f(x)=x+g(x)$ for all $x \geq 0$. Then $f$ is metric preserving if and only iff is increasing and subadditive. 
Proof The proof can be found in $[8, \mathrm{p} .32]$ and $[16$, Theorem 1$]$.

Lemma 13 (Hu and Kirk [22]) Let $(X, d)$ be a complete metric space for which each two points can be joined by a rectifiable path, and suppose $g: X \rightarrow X$ is a local radial contraction. Then $g$ has a unique fixed point $x_{0}$, and $\lim _{n \rightarrow \infty} g^{n}(x)=x_{0}$ for each $x \in X$.

As noted by Kirk and Shahzad [21], an assertion in the proof of Lemma 13 given in [22] was based on a false proposition of Holmes [23]. But Jungck [24] proved that the assertion itself is true. Hence the proof given in [22] with minor changes is true. Kirk and Shahzad [21] apply Tan's result [25] to extend some of their theorems. We will also apply Tan's result as well.

Lemma 14 (Tan [25]) Let $X$ be a topological space, let $x_{0} \in X$, and let $g: X \rightarrow X$ be a mapping for which $f:=g^{N}$ satisfies $\lim _{n \rightarrow \infty} f^{n}(x)=x_{0}$ for each $x \in X$. Then $\lim _{n \rightarrow \infty} g^{n}(x)=$ $x_{0}$ for each $x \in X$. (Also if $x_{0}$ is the unique fixed point of $f$, it is also the unique fixed point of $g$.)

We will use Nadler's result concerning set-valued mappings. So let us recall some more definitions. If $\varepsilon>0$ is given, a metric space $(X, d)$ is said to be $\varepsilon$-chainable if given $a, b \in X$ there exist $x_{1}, x_{2}, \ldots, x_{n} \in X$ such that $a=x_{1}, b=x_{n}$, and $d\left(x_{i}, x_{i+1}\right)<\varepsilon$ for all $i \in\{1,2, \ldots, n-$ $1\}$. The result of Nadler that we need is the following.

Lemma 15 (Nadler [26]) Let $(X, d)$ be a complete $\varepsilon$-chainable metric space. If $T: X \rightarrow$ $\mathcal{C B}(X)$ is an $(\varepsilon, k)$-uniform local multivalued contraction, then $T$ has a fixed point.

\section{Local radial contractions and metric-preserving functions}

In this section, we will give a generalization of Theorem 1 where the metric transform $\phi$ is replaced by a metric-preserving function. In fact, we obtain a more general result as follows.

Theorem 16 Let $(X, d)$ be a metric space and let $g: X \rightarrow X$. Assume that there exist $k \in$ $(0,1)$ and a metric-preserving function $f$ satisfying the following conditions:

(a) for each $x \in X$, there exists $\varepsilon>0$ such that for every $u \in X$

$$
d(x, u)<\varepsilon \quad \Rightarrow \quad(f \circ d)(g(x), g(u)) \leq k d(x, u), \quad \text { and }
$$

(b) $f^{\prime}(0)>k$.

Then $g$ is a local radial contraction.

We know from Lemma 10 that $f^{\prime}(0)$ always exists in $\mathbb{R} \cup\{+\infty\}$. So condition (b) in Theorem 16 makes sense. To prove this theorem, we will first show that $g$ is continuous in the following lemma.

Lemma 17 Suppose that the assumptions in Theorem 16 hold. Then the function $g$ is continuous.

As a consequence of Theorem 16, we can replace the metric transform $\phi$ in Theorem 1 by a metric-preserving function and obtain an extension of Theorem 1. 
Theorem 18 With the same assumptions in Theorem 16 except that condition (b) is replaced by $\left(\mathrm{b}^{\prime}\right)$ : there exists $c \in(0,1)$ such that $f(c t) \geq k t$ for all $t>0$ sufficiently small. Then $g$ is a local radial contraction.

Remark 19 As noted by Kirk and Shahzad [21, Remark 2.5], [21, Proposition 2.6], metric transforms satisfying condition (b) in Theorem 1 are numerous. Proposition 20, Example 22, and Example 23 (to be given after the proof of Theorem 18) show that the class of metric-preserving functions satisfying condition (b) in Theorem 1 is larger than the class of metric transforms satisfying the same condition. Hence the class of such functions is even more numerous and Theorem 18 is indeed an extension of Theorem 1.

Now let us give the proof of Lemma 17, Theorem 16, and Theorem 18 as follows.

Proof of Lemma 17 Let $x \in X$ and let $\varepsilon>0$. Since $k<f^{\prime}(0)=\lim _{y \rightarrow 0^{+}} \frac{f(y)-f(0)}{y-0}=\lim _{y \rightarrow 0^{+}} \frac{f(y)}{y}$, there exists $\delta_{1}>0$ such that

$$
0<y \leq \delta_{1} \quad \Rightarrow \quad \frac{f(y)}{y}>k
$$

By condition (a), there exists $\delta_{2}>0$ such that for every $u \in X$,

$$
d(x, u)<\delta_{2} \quad \Rightarrow \quad(f \circ d)(g(x), g(u)) \leq k d(x, u) .
$$

Let $\delta_{3}=\min \left\{\delta_{1}, \delta_{2}, \varepsilon\right\}$. Then by (1), we obtain

$$
\frac{f\left(\delta_{3}\right)}{\delta_{3}}>k
$$

Since $f$ is metric preserving, we obtain by Lemma 9 , and (3) that for every $b \in[0, \infty)$

$$
b \geq \frac{\delta_{3}}{2} \Rightarrow f(b) \geq \frac{f\left(\delta_{3}\right)}{2}>\frac{k \delta_{3}}{2} .
$$

Now let $\delta=\frac{\delta_{3}}{2}$ and $u \in X$ be such that $d(x, u)<\delta$. Then by (2), we obtain

$$
f(d(g(x), g(u))) \leq k d(x, u)<k \delta=\frac{k \delta_{3}}{2} .
$$

Then by (4), $d(g(x), g(u))<\frac{\delta_{3}}{2} \leq \frac{\varepsilon}{2}<\varepsilon$. This shows that $g$ is continuous, as required.

Proof of Theorem 16 Let $c=\frac{1}{2}\left(\frac{k}{f^{\prime}(0)}+1\right)$ where if $f^{\prime}(0)=+\infty$, we define $\frac{k}{f^{\prime}(0)}$ to be zero and $c=\frac{1}{2}(0+1)=\frac{1}{2}$. Then $0 \leq \frac{k}{f^{\prime}(0)}<c<1$. Consider

$$
f^{\prime}(0)=\lim _{y \rightarrow 0^{+}} \frac{f(y)-f(0)}{y-0}=\lim _{y \rightarrow 0^{+}} \frac{f(y)}{y} .
$$

Since $f^{\prime}(0)>\frac{k}{c}$, there exists $\delta_{1}>0$ such that

$$
0<y<\delta_{1} \Rightarrow \frac{f(y)}{y}>\frac{k}{c} .
$$


To show that $g$ is a local radial contraction with the contraction constant $c$, let $x \in X$. By Lemma 17, $g$ is continuous at $x$. So there exists $\delta_{2}>0$ such that for every $u \in X$,

$$
d(x, u)<\delta_{2} \quad \Rightarrow \quad d(g(x), g(u))<\delta_{1}
$$

By condition (a), there exists $\delta_{3}>0$ such that for every $u \in X$,

$$
d(x, u)<\delta_{3} \quad \Rightarrow \quad(f \circ d)(g(x), g(u)) \leq k d(x, u) .
$$

Now let $\varepsilon=\min \left\{\delta_{1}, \delta_{2}, \delta_{3}\right\}$ and let $u \in X$ be such that $d(x, u)<\varepsilon$. We need to show that $d(g(x), g(u)) \leq c d(x, u)$. If $d(g(x), g(u))=0$, then we are done. So assume that $d(g(x), g(u))>$ 0 . Then $0<d(x, u)<\varepsilon$ and we obtain by $(7)$ that

$$
\frac{(f \circ d)(g(x), g(u))}{d(x, u)} \leq k
$$

The left hand side of (8) is

$$
\begin{aligned}
\frac{(f \circ d)(g(x), g(u))}{d(x, u)} & =\frac{f(d(g(x), g(u)))}{d(g(x), g(u))} \cdot \frac{d(g(x), g(u))}{d(x, u)} \\
& >\frac{k}{c} \frac{d(g(x), g(u))}{d(x, u)}
\end{aligned}
$$

where the above inequality is obtained from (6) and (5). From (8) and (9), we obtain

$$
\frac{k}{c} \frac{d(g(x), g(u))}{d(x, u)}<k
$$

which implies the desired result. This completes the proof.

Proof of Theorem 18 By Lemma 10, we know that $f^{\prime}(0)$ exists in $\mathbb{R} \cup\{+\infty\}$ and by Theorem 16, it suffices to show that $f^{\prime}(0)>k$. So we can assume further that $f^{\prime}(0)$ exists in $\mathbb{R}$. Now $f^{\prime}(0)=\lim _{y \rightarrow 0^{+}} \frac{f(y)-f(0)}{y-0}=\lim _{y \rightarrow 0^{+}} \frac{f(y)}{y}$. Since the limits involved in the following calculation exist, we obtain

$$
\lim _{y \rightarrow 0^{+}} \frac{f(y)}{y}=\lim _{t \rightarrow 0^{+}} \frac{f(c t)}{c t} \geq \lim _{t \rightarrow 0^{+}} \frac{k t}{c t}=\frac{k}{c}>k
$$

Therefore $f^{\prime}(0)>k$, as desired.

As noted earlier, we will show that the class of metric-preserving functions and the class of metric-preserving functions satisfying condition (b) in Theorem 1 are, respectively, larger than the class of metric transforms and the class of metric transforms satisfying condition (b) in Theorem 1.

Proposition 20 Every metric transform is metric preserving.

Proof Let $f$ be a metric transform. Since $f(0)=0$ and $f$ is strictly increasing, $f$ is amenable. Since $f$ is amenable and concave, we obtain by Lemma 6(ii) that $f$ is metric preserving. 
Corollary 21 Kirk and Shahzad's result (Theorem 1) holds.

Proof This follows immediately from Proposition 20 and Theorem 18.

Example 22 Let $f, g, h:[0, \infty) \rightarrow[0, \infty)$ be given by



$$
\begin{aligned}
& h(x)= \begin{cases}x, & x \in[0,1] ; \\
1, & x \in[1,10] ; \\
x-9, & x \in(10,11) ; \\
2, & x \geq 11 .\end{cases}
\end{aligned}
$$

Since $f(x) \in[1,2]$ for all $x>0, f$ is tightly bounded. Therefore by Lemma $8, f$ is metric preserving. It is easy to see that $f$ is not increasing (and is not concave either). So $f$ is not a metric transform. It is easy to see that $g$ is amenable and concave, so it is metric preserving, by Lemma 6(ii). In addition, if $c=k=\frac{1}{2} \in(0,1)$, then $g(c t) \geq k t$ for all $t \in[0,1]$. So $g$ satisfies condition (b) in Theorem 1. But $g$ is not a metric transform because it is not strictly increasing. For $h$, we proved in [27, Example 14] that $h$ is metric preserving. Similar to $g$, the function $h$ satisfies the condition (b) in Theorem 1 . It is easy to see that $h$ is neither strictly increasing nor concave. Therefore $h$ is not a metric transform.

We can generate more functions similar to $g$ given in Example 22 as follows.

Example 23 Let $a \geq 1$ and $b>0$. Define $f_{a, b}:[0, \infty) \rightarrow[0, \infty)$ by

$$
f_{a, b}(x)= \begin{cases}a x, & \text { if } x \in[0, b] \\ a b, & \text { if } x>b\end{cases}
$$

Then $f_{a, b}$ is amenable and concave. So by Lemma 6(ii), $f_{a, b}$ is metric preserving. We also have $f_{a, b}^{\prime}(0)=a \geq 1$. So it satisfies condition (b) in Theorem 16 . However, $f_{a, b}$ is not a metric transform because it is not strictly increasing. In particular, if we let $X=[0, \infty), k=\frac{1}{2}, f, g$ : $X \rightarrow X$ given by $g(x)=\frac{1}{2} x$ and $f=f_{1,1}$, then $f$ satisfies all the assumptions in Theorem 16 .

Remark 24 Some natural questions concerning the relation of metric transforms, metricpreserving functions, and condition (b) can be answered by Example 22 and Example 23:

Q1: Is there a continuous metric-preserving function which is not a metric transform?

A1: Yes, $g$ and $h$ given in Example 22 and $f_{a, b}$ given in Example 23 are such functions.

Q2: Is there any nowhere continuous metric-preserving function which is not a metric transform?

A2: Yes, $f$ given in Example 22 is such a function.

Q3: Is there a nowhere monotone metric-preserving function which is not a metric transform?

A3: Yes, $f$ given in Example 22 is such a function. 
Q4: Is there a metric-preserving function which is concave and satisfies condition (b) in Theorem 1 but it is not a metric transform?

A4: Yes, $g$ given in Example 22 and $f_{a, b}$ given in Example 23 are such functions.

Now that we have obtained two extensions of Theorem 1, we give two generalizations of Theorem 2 as follows.

Theorem 25 The following statements hold:

(a) Suppose, in addition to the assumptions in Theorem 16, $X$ is complete and rectifiably pathwise connected. Then $g$ has a unique fixed point $x_{0}$, and $\lim _{n \rightarrow \infty} g^{n}(x)=x_{0}$ for each $x \in X$.

(b) Suppose, in addition to the assumptions in Theorem 18, $X$ is complete and rectifiably pathwise connected. Then $g$ has a unique fixed point $x_{0}$, and $\lim _{n \rightarrow \infty} g^{n}(x)=x_{0}$ for each $x \in X$.

Proof Part (a) follows immediately from Theorem 16 and Lemma 13. Part (b) follows immediately from Theorem 18 and Lemma 13.

Finally, we remark that Kirk and Shahzad use Tan's result (Lemma 14) to extend Theorem 2 further [21, Theorem 2.3 and Theorem 2.8]. We similarly apply their argument to obtain the following.

Theorem 26 Let $X$ be a metric space which is complete and rectifiably pathwise connected, and suppose $g: X \rightarrow X$ is a mapping for which

(a) $g^{N}$ satisfies the assumptions in Theorem 16 for some $N \in \mathbb{N}$, or

(b) $g^{M}$ satisfies the assumptions in Theorem 18 for some $M \in \mathbb{N}$.

Then $g$ has a unique fixed point $x_{0}$, and $\lim _{n \rightarrow \infty} g^{n}(x)=x_{0}$ for each $x \in X$.

Proof This follows immediately from Theorem 16, Theorem 18, Lemma 13, and Lemma 14.

Conclusion We have obtained extensions of the main results given by Kirk and Shahzad in [21, Theorem 2.2, Theorem 2.3, and Theorem 2.8]. We will obtain more results in the next section.

\section{Set-valued contractions}

Kirk and Shahzad [21] also give an analog of Theorem 1 and Theorem 2 for set-valued mappings. Our purpose in this section is to obtain an analog of Theorem 16 and Theorem 18 for set-valued mappings as well. First let us recall some definitions and results concerning set-valued mappings.

Let $(X, d)$ be a metric space and let $\mathcal{C B}(X)$ be the family of nonempty, closed, and bounded subsets of $X$. The usual Hausdorff distance on $\mathcal{C B}(X)$ is defined as

$$
H(A, B)=\max \{\rho(A, B), \rho(B, A)\},
$$

where $A, B \in \mathcal{C B}(X), \rho(A, B)=\sup _{x \in A} d(x, B), \rho(B, A)=\sup _{x \in B} d(x, A)$.

Definition 27 Let $T: X \rightarrow \mathcal{C B}(X)$. Then 
(i) $T$ is called a multivalued contraction mapping if there exists a constant $k \in(0,1)$ such that $H(T x, T y) \leq k d(x, y)$ for all $x, y \in X$.

(ii) For $\varepsilon>0$ and $k \in(0,1), T$ is called an $(\varepsilon, k)$-uniform local multivalued contraction if for every $x, y \in X$

$$
d(x, y)<\varepsilon \quad \Rightarrow \quad H(T x, T y) \leq k d(x, y) .
$$

(iii) A point $x \in X$ is said to be a fixed point of $T$ if $x \in T x$.

Kirk and Shahzad's results on set-valued mappings which will be extended are as follows.

Theorem 28 (Kirk and Shahzad [21, Theorem 3.4]) Let $(X, d)$ be a metric space and $T$ : $X \rightarrow \mathcal{C B}(X)$. Suppose there exist a metric transform $\phi$ and $k \in(0,1)$ such that the following conditions hold:

(a) For each $x, y \in X, \phi(H(T x, T y)) \leq k d(x, y)$.

(b) There exists $c \in(0,1)$ such that for $t>0$ sufficiently small, $k t \leq \phi(c t)$.

Then for $\varepsilon>0$ sufficiently small, $T$ is an $(\varepsilon, c)$-uniform local multivalued contraction on $(X, d)$.

Theorem 29 (Kirk and Shahzad [21, Theorem 3.6]) If, in addition to the assumptions of Theorem 28, $X$ is complete and connected, then $T$ has a fixed point.

Our aim is to replace the metric transform $\phi$ in Theorem 28 by a metric-preserving function. We obtain the following theorem.

Theorem 30 Let $(X, d)$ be a metric space and $T: X \rightarrow \mathcal{C B}(X)$. Suppose there exist a metricpreserving function $f$ and $k \in(0,1)$ such that the following conditions hold:

(a) For each $x, y \in X, f(H(T x, T y)) \leq k d(x, y)$.

(b) $f^{\prime}(0)>k$.

Then for $\varepsilon>0$ sufficiently small, $T$ is an $(\varepsilon, c)$-uniform local multivalued contraction on $(X, d)$.

Corollary 31 With the same assumptions in Theorem 30 except that condition (b) is replaced by $\left(\mathrm{b}^{\prime}\right)$ : there exists $c \in(0,1)$ such that for $t>0$ sufficiently small, $k t \leq f(c t)$. Then for $\varepsilon>0$ sufficiently small, $T$ is an $(\varepsilon, c)$-uniform local multivalued contraction on $(X, d)$.

Theorem 32 If, in addition to the assumptions of Theorem 30 or Corollary 31, $X$ is complete and $\varepsilon$-chainable, then $T$ has a fixed point. In particular, if $X$ is complete and connected, then $T$ has a fixed point.

The proof of these results are similar to those in Section 3.

Proof of Theorem 30 We define $c=\frac{1}{2}\left(\frac{k}{f^{\prime}(0)}+1\right)$ as in the proof of Theorem 16. Then $0 \leq$ $\frac{k}{f^{\prime}(0)}<c<1$ and there exists $\delta_{1}>0$ such that for every $z \in[0, \infty)$

$$
0<z \leq \delta_{1} \Rightarrow \frac{f(z)}{z}>\frac{k}{c}
$$


To show that $T$ is an $(\varepsilon, c)$-uniform local multivalued contraction for $\varepsilon>0$ sufficiently small, we let $0<\varepsilon<\frac{\delta_{1}}{2}$ and let $x, y \in X$ be such that $d(x, y)<\varepsilon$. By Lemma 9 and (10), we have for every $b \in[0, \infty)$

$$
b \geq \frac{\delta_{1}}{2} \Rightarrow f(b) \geq \frac{f\left(\delta_{1}\right)}{2}>\frac{k \delta_{1}}{2 c}>\frac{k \varepsilon}{c}>k \varepsilon .
$$

By condition (a), we have $f(H(T x, T y)) \leq k d(x, y)<k \varepsilon$. Therefore we obtain by (11) that

$$
H(T x, T y)<\frac{\delta_{1}}{2}
$$

If $d(x, y)=0$ or $H(T x, T y)=0$, then it is obvious that $H(T x, T y) \leq c d(x, y)$ and we are done. So assume that $H(T x, T y)>0$ and $d(x, y)>0$. Then

$$
\frac{k}{c} \frac{H(T x, T y)}{d(x, y)}<\frac{f(H(T x, T y))}{H(T x, T y)} \cdot \frac{H(T x, T y)}{d(x, y)}=\frac{f(H(T x, T y))}{d(x, y)} \leq k,
$$

where the first inequality is obtained by applying (12) and (10) and the last inequality is merely the condition (a). This implies $H(T x, T y) \leq c d(x, y)$, as desired.

Proof of Corollary 31 We can imitate the proof of Theorem 18 to obtain $f^{\prime}(0)>k$. So Corollary 31 follows immediately from Theorem 30 .

Proof of Theorem 32 This follows from Theorem 30, Corollary 31, and Lemma 15. The other part follows from the fact that a connected metric space is $\varepsilon$-chainable for every $\varepsilon>0$.

Conclusion We replace the metric transform $\phi$ by a metric-preserving function. Therefore we obtain theorems more general than those of Kirk and Shahzad [21, Theorem 2.2, Theorem 2.3, Theorem 2.8, Theorem 3.4, and Theorem 3.6].

\section{Fixed point set of metric transforms and metric-preserving functions}

Recall that for a function $f: X \rightarrow X$, we denote by Fix $f$ the set of all fixed points of $f$. We begin this section with the following lemma.

Lemma 33 Let $f:[0, \infty) \rightarrow[0, \infty)$ be a metric transform. If $0<a<b, f(a)=a$, and $f(b)=$ $b$, then $[a, b] \subseteq \operatorname{Fix} f$.

Proof Since $f$ is amenable and concave, the function $x \mapsto \frac{f(x)}{x}$ is decreasing on $(0, \infty)$ by Lemma 11. So if $a \leq x \leq b$, then $1=\frac{f(a)}{a} \geq \frac{f(x)}{x} \geq \frac{f(b)}{b}=1$, which implies $f(x)=x$. This shows that $[a, b] \subseteq \operatorname{Fix} f$.

Lemma 34 If $f:[0, \infty) \rightarrow[0, \infty)$ is a metric transform, then $\operatorname{Fix} f$ is a closed subset of $[0, \infty)$

Proof Let $\left(a_{n}\right)$ be a sequence in Fix $f$ and $a_{n} \rightarrow a$. If $a=0$ or $a=a_{n}$ for some $n \in \mathbb{N}$, then $a \in \operatorname{Fix} f$ and we are done. So assume that $a>0$ and $a \neq a_{n}$ for any $n \in \mathbb{N}$. Since $a>0$ and $a_{n} \rightarrow a, a_{n}>0$ for all large $n$. By passing to the subsequence, we can assume that $a_{n}>0$ 
for every $n \in \mathbb{N}$. It is well known that every sequence of real numbers has a monotone subsequence (see e.g. [28, p.62]). By passing to the subsequence again, we can assume that $\left(a_{n}\right)$ is monotone. Now suppose that $\left(a_{n}\right)$ is increasing. Then by Lemma 33,

$$
\left[a_{1}, a_{n}\right] \subseteq\left[a_{1}, a_{2}\right] \cup\left[a_{2}, a_{3}\right] \cup \cdots \cup\left[a_{n-1}, a_{n}\right] \subseteq \text { Fix } f \quad \text { for every } n \in \mathbb{N}
$$

Since $\left(a_{n}\right)$ is increasing and $a_{n} \rightarrow a$, if $a_{1} \leq x<a$, then there exists $N \in \mathbb{N}$ such that $a_{1} \leq$ $x<a_{N}$, which implies that $x \in \operatorname{Fix} f$, by Lemma 33. This shows that $\left[a_{1}, a\right) \subseteq \operatorname{Fix} f$. Since $f$ is increasing and $a_{n}<a, a_{n}=f\left(a_{n}\right) \leq f(a)$ for every $n \in \mathbb{N}$. Since $a_{n} \leq f(a)$ for every $n \in \mathbb{N}$ and $a_{n} \rightarrow a$, we have

$$
a \leq f(a) .
$$

In addition, we obtain by Lemma 11 and the fact that $a \geq a_{1}$ that

$$
\frac{f(a)}{a} \leq \frac{f\left(a_{1}\right)}{a_{1}}=1 .
$$

From (13) and (14), we obtain $f(a)=a$, as required. The case where $\left(a_{n}\right)$ is decreasing can be proved similarly. This completes the proof.

Lemma 35 Let $f:[0, \infty) \rightarrow[0, \infty)$ be a metric transform. Then $\operatorname{Fix} f=[0, \infty)$ if and only if $\sup \operatorname{Fix} f=+\infty$.

Proof It is enough to show that $\sup \operatorname{Fix} f=+\infty$ implies $(0, \infty) \subseteq \operatorname{Fix} f$. So suppose that $\sup$ Fix $f=+\infty$ but there exists $x \in(0, \infty)$ such that $f(x) \neq x$. Since $\sup$ Fix $f=+\infty$, there exists $a>x$ such that $f(a)=a$. Similarly, there exists $b>a$ such that $f(b)=b$. Since $f$ is amenable and concave, we obtain by Lemma 11

$$
\frac{f(x)}{x} \geq \frac{f(a)}{a}=1 .
$$

Since $f(x) \neq x, f(x)>x$. Since $x<a<b$, there exists $t \in(0,1)$ such that $a=(1-t) x+t b$. By the concavity of $f$, we obtain

$$
a=f(a)=f((1-t) x+t b) \geq(1-t) f(x)+t f(b)>(1-t) x+t b=a,
$$

a contradiction. This completes the proof.

Theorem 36 If $a>0$, then each set of the form $\{0\},\{0, a\},[0, a]$, and $[0, \infty)$ is a fixed point set of a metric transform. Conversely, iff is a metric transform, then $\operatorname{Fix} f=\{0\},\{0, a\},[0, a]$, or $[0, \infty)$ for some $a \in(0, \infty)$.

Proof Define $f_{1}, f_{2}, f_{3}, f_{4}:[0, \infty) \rightarrow[0, \infty)$ by

$$
f_{1}(x)=\frac{x}{2}, \quad f_{2}(x)=\sqrt{a x}, \quad f_{3}(x)=x, \quad f_{4}(x)= \begin{cases}x, & x \in[0, a] \\ \frac{x+a}{2}, & x>a\end{cases}
$$


It is easy to verify that the functions $f_{1}, f_{2}, f_{3}, f_{4}$ are metric transforms and Fix $f_{1}=\{0\}$, $\operatorname{Fix} f_{2}=\{0, a\}, \operatorname{Fix} f_{3}=[0, \infty)$, and $\operatorname{Fix} f_{4}=[0, a]$. This proves the first part.

Next let $f$ be a metric transform such that Fix $f \neq\{0\}$ and Fix $f \neq[0, \infty)$. We let $a=$ $\sup \operatorname{Fix} f$ and assert that $\operatorname{Fix} f=\{0, a\}$ or $[0, a]$. Note that since $\operatorname{Fix} f \neq\{0\}, a>0$. It is obtained by Lemma 35 that $a<+\infty$. Now apply Lemma 34 to get $a \in \operatorname{Fix} f$. Therefore $\{0, a\} \subseteq \operatorname{Fix} f$. By the definition of $a$, we see that $x \notin \operatorname{Fix} f$ for every $x>a$. Now if $x \notin \operatorname{Fix} f$ for every $0<x<a$, then $\operatorname{Fix} f=\{0, a\}$ and we are done. So assume that there exists $0<x<a$ such that $x \in \operatorname{Fix} f$. We will show that $\operatorname{Fix} f=[0, a]$. Since $a=\sup \operatorname{Fix} f$, it is obvious that Fix $f \subseteq[0, a]$. Suppose for a contradiction that there exists $0<y<a$ such that $f(y) \neq y$. Since $0<x<a$ and $x, a \in \operatorname{Fix} f$, we obtain by Lemma 33 that $y \notin[x, a]$. So $y<x$. By Lemma 11 we have

$$
\frac{f(y)}{y} \geq \frac{f(x)}{x}=1 .
$$

Since $f(y) \neq y, f(y)>y$. Since $y<x<a$, there exists $t \in(0,1)$ such that $x=(1-t) y+t a$. By the concavity of $f$, we obtain

$$
x=f(x)=f((1-t) y+t a) \geq(1-t) f(y)+t f(a)>(1-t) y+t a=x,
$$

a contradiction. This completes the proof.

Since every metric transform is metric preserving, we immediately obtain the result that each set of the form $\{0\},\{0, a\},[0, a]$, and $[0, \infty)$ is a fixed point set of a metric-preserving function. However, there is a metric-preserving function $f$ where $\operatorname{Fix} f$ is not of this form. Let us show this more precisely.

Corollary 37 If $a>0$, then each set of the form $\{0\},\{0, a\},[0, a]$, and $[0, \infty)$ is a fixed point of a metric-preserving function.

Proof This follows immediately from Theorem 36 and Proposition 20.

Example 38 Let $f, g, h:[0, \infty) \rightarrow[0, \infty)$ be given by

$$
\begin{aligned}
& f(x)=\lceil x\rceil, \quad g(x)= \begin{cases}0, & x=0 ; \\
1, & x \in \mathbb{Q}-\{0\} ; \\
\sqrt{2}, & x \in \mathbb{Q}^{c},\end{cases} \\
& h(x)= \begin{cases}0, & x=0 ; \\
1, & 0<x<1 ; \\
x, & x \in \mathbb{Q} \cap[1,2] ; \\
2, & x \in\left(\mathbb{Q}^{c} \cap[1,2]\right) \cup(2, \infty) .\end{cases}
\end{aligned}
$$

(Recall that $\lceil x\rceil$ is the smallest integer which is larger than or equal to $x$.) It is easy to verify that $f$ is amenable, increasing, and subadditive. So by Lemma $7, f$ is metric preserving. Since $g$ and $h$ are amenable and tightly bounded, we obtain by Lemma 8 that $g$ and $h$ are metric-preserving. It is easy to see that $\operatorname{Fix} f=\mathbb{N} \cup\{0\}$, Fix $g=\{0,1, \sqrt{2}\}$, and Fix $h=$ $\{0\} \cup(\mathbb{Q} \cap[1,2])$. 
By generating a function similar to $h$ we obtain a more general result as follows.

Proposition 39 Let $A \subseteq[u, 2 u]$ for some $u>0$. Then $A \cup\{0\}$ is a fixed point set of a metricpreserving function.

Proof We define $f:[0, \infty) \rightarrow[0, \infty)$ by

$$
f(x)= \begin{cases}0, & \text { if } x=0 ; \\ x, & \text { if } x \in A ; \\ u, & \text { if } x \notin A \wedge x \notin\{0, u\},\end{cases}
$$

and if $u \notin A$, then define $f(u)=2 u$. Then $f$ is amenable and tightly bounded. Therefore, by Lemma $8, f$ is metric preserving. It is easy to see that Fix $f=A \cup\{0\}$. This completes the proof.

From Example 38 and Proposition 39, we see that the fixed point set of a metricpreserving function may not be of the form $\{0\},\{0, a\},[0, a]$, and $[0, \infty)$. Other natural questions and answers are the following:

Q1: Is there a metric-preserving function which does not satisfy the result in Lemma 33?

A1: Every function given in Example 38 is such a function.

Q2: Is there a metric-preserving function which does not satisfy the result in Lemma 34 ?

A2: The function $h$ given in Example 38 and the function $f$ given in Proposition 39 (with a suitable set $A$ ) are such functions.

Q3: Is there a metric-preserving function which does not satisfy the result in Lemma 35 ?

A3: The function $f$ given in Example 38 is such a function.

We see that the fixed point sets of metric-preserving functions are quite difficult to be completely characterized. We leave this problem to the interested reader. Now we end this article by giving continuous metric-preserving functions which do not satisfy the results in Lemma 33 and Lemma 35.

Example 40 Let $f, g:[0, \infty) \rightarrow[0, \infty)$ be given by $f(x)=\lfloor x\rfloor+\sqrt{x-\lfloor x\rfloor}$ and $g(x)=x+$ $|\sin x|$. (Recall that $\lfloor x\rfloor$ is the largest integer which is less than or equal to $x$.) We will use Lemma 12 to show that $f$ and $g$ are metric-preserving. First, the function $x \mapsto|\sin x|$ is periodic with period $\pi$.

$$
|\sin (x+y)|=|\sin x \cos y+\cos x \sin y| \leq|\sin x|+|\sin y| .
$$

So the function $x \mapsto|\sin x|$ is also subadditive. From this, we easily see that $g$ satisfies the condition in Lemma 12. So $g$ is metric preserving. It is not difficult to verify that $f$ is also satisfies the assumption in Lemma 12 and we will leave the details to the reader. It is also easy to see that Fix $f=\mathbb{N} \cup\{0\}$ and Fix $g=\{n \pi \mid n \in \mathbb{N} \cup\{0\}\}$. So $f$ and $g$ are continuous metric preserving functions of which fixed point sets do not satisfy the results in Lemma 33 and Lemma 35. 


\section{Competing interests}

The authors declare that they have no competing interests.

\section{Authors' contributions}

All authors contributed significantly in writing this paper. All authors read and approved this final manuscript.

\section{Author details}

${ }^{1}$ Department of Mathematics, Faculty of Science, Silpakorn University, Nakhon Pathom, 73000, Thailand. ${ }^{2}$ Department of Mathematics and Computer Science, Faculty of Science, Chulalongkorn University, Bangkok, 10330, Thailand.

\section{Acknowledgements}

The first author would like to thank The Thailand Research Fund for financial support under the contract number TRG5680052. Both authors also would like to thank Professor IA Rus for sending them his article on the role of equivalent metrics and metric-preserving functions in fixed point theory which helps improve the presentation of this article.

Received: 31 January 2014 Accepted: 4 August 2014 Published: 01 Sep 2014

\section{References}

1. Blumenthal, LM: Theory and Applications of Distance Geometry, 2nd edn. Chelsea, New York (1970)

2. Blumenthal, LM: Remarks concerning the Euclidean four-point property. Ergebnisse Math. Kolloq. Wien 7, 7-10 (1936)

3. Wilson, WA: On certain types of continuous transformations of metric spaces. Am. J. Math. 57, 62-68 (1935)

4. Borsík, J, Doboš, J: On metric preserving functions. Real Anal. Exch. 13, 285-293 (1987/1988)

5. Borsík, J, Doboš, J: Functions whose composition with every metric is a metric. Math. Slovaca 31, 3-12 (1981)

6. Corazza, P: Introduction to metric-preserving functions. Am. Math. Mon. 106(4), 309-323 (1999)

7. Das, PP: Metricity preserving transforms. Pattern Recognit. Lett. 10, 73-76 (1989)

8. Doboš, J: Metric Preserving Functions. Online Lecture Notes. Available at http://web.science.upjs.sk/jozefdobos/wp-content/uploads/2012/03/mpf1.pdf

9. Doboš, J: On modification of the Euclidean metric on reals. Tatra Mt. Math. Publ. 8, 51-54 (1996)

10. Doboš, J: A survey of metric-preserving functions. Quest. Answ. Gen. Topol. 13, 129-133 (1995)

11. Doboš, J, Piotrowski, Z: When distance means money. Int. J. Math. Educ. Sci. Technol. 28, 513-518 (1997)

12. Doboš, J, Piotrowski, Z: A note on metric-preserving functions. Int. J. Math. Math. Sci. 19, 199-200 (1996)

13. Doboš, J, Piotrowski, Z: Some remarks on metric-preserving functions. Real Anal. Exch. 19, 317-320 (1993/1994)

14. Petruşel, A, Rus, IA, Şerban, MA: The role of equivalent metrics in fixed point theory. Topol. Methods Nonlinear Anal. 41(1), 85-112 (2013)

15. Piotrowski, Z, Vallin, RW: Functions which preserve Lebesque spaces. Comment. Math. Prace Mat. 43(2), 249-255 (2003)

16. Pokorný, I: Some remarks on metric-preserving functions. Tatra Mt. Math. Publ. 2, 65-68 (1993)

17. Pokorný, I: Some remarks on metric-preserving functions of several variables. Tatra Mt. Math. Publ. 8, 89-92 (1996)

18. Sreenivasan, TK: Some properties of distance functions. J. Indian Math. Soc. 11, 38-43 (1947)

19. Termwuttipong, I, Oudkam, P: Total boundedness, completeness and uniform limits of metric-preserving functions. Ital. J. Pure Appl. Math. 18, 187-196 (2005)

20. Vallin, RW: Continuity and differentiability aspects of metric preserving functions. Real Anal. Exch. 25(2), 849-868 (1999/2000)

21. Kirk, WA, Shahzad, N: Remarks on metric transforms and fixed-point theorems. Fixed Point Theory Appl. 2013, 106 (2013)

22. Hu, T, Kirk, WA: Local contractions in metric spaces. Proc. Am. Math. Soc. 68, 121-124 (1978)

23. Holmes, RD: Fixed points for local radial contractions. In: Swaminathan, S (ed.) Fixed Point Theory and Its Applications, pp. 79-89. Academic Press, New York (1976)

24. Jungck, G: Local radial contractions - a counter - example. Houst. J. Math. 8, 501-506 (1982)

25. Tan, KK: Fixed point theorems for nonexpansive mappings. Pac. J. Math. 41, 829-842 (1972)

26. Nadler, SB Jr: Multi-valued contraction mappings. Pac. J. Math. 30, 475-488 (1969)

27. Pongsriiam, $P$, Termwuttipong, I: Remarks on ultrametrics and metric-preserving functions. Abstr. Appl. Anal. 2014, Article ID 163258 (2014)

28. Thomson, BS, Bruckner, JB, Bruckner, AM: Elementary Real Analysis. Prentice Hall, New York (2001)

10.1186/1687-1812-2014-179

Cite this article as: Pongsriiam and Termwuttipong: On metric-preserving functions and fixed point theorems. Fixed Point Theory and Applications 2014, 2014:179 